Revista Científica de FAREM-Estelí

Medio ambiente. tecnología y desarrollo humano

Año 10 | Edición especial: artículos de revisión documental

ISSN: 2305-5790

https://rcientificaesteli.unan.edu.ni

DOI: https://doi.org/10.5377/farem.v0i0.11604

\section{Elementos conceptuales para la construcción histórica de una localidad}

\section{Conceptual Elements for the historical construction of a locality}

\section{Pablo José Escalante Turcios}

Docente de la Universidad Nacional Autónoma de Nicaragua. UNAN. León. Estudiante de doctorado en Gestión y Calidad de la Investigación Científica. II Edición. Universidad Nacional Autónoma de Nicaragua, UNAN-Managua, Facultad Regional Multidisciplinaria de Estelí (FAREM-Estelí). II Cohorte. 20202023.

https://orcid.org/0000-0001-8650-0097

escalpjet@gmail.com

\section{RESUMEN}

El presente trabajo tiene por objetivo revisar los elementos conceptuales que sustenten la construcción histórica de una localidad; mediante las teorías relacionadas a la microhistoria y redes sociales. En la metodología se procedió a la consulta del repositorio del Consejo Nacional de Universidades, referida a libros, tesis doctorales y artículos vinculados al objeto de estudio; agotado este recurso se procedió a la indagación en Google académico. Para cada tópico se exploraron varios títulos, se retomaron las ideas relacionadas a la temática, se hizo análisis de la información; por autor y por conceptos Se seleccionaron las ideas que sirviesen de fundamento al problema de investigación. Se concluye que para la construcción de la historia de una localidad se deben integrar varios componentes del método bibliográfico, tales como: la historia regional y local, memoria histórica, redes sociales, historia de vidas y oral, para el rescate de hechos, acontecimientos y sus significados, de un periodo determinado de una sociedad en un territorio. No solo se trata de los saberes acerca del pasado, sino que también implica la organización de las personas para que desde su individualidad formen parte de un pensamiento colectivo.
RECIBIDO

$26 / 03 / 2021$

ACEPTADO

19/052021
PALABRAS CLAVE

Historia regional y local; memoria histórica; historia oral; redes sociales. 


\section{ABSTRACT}

The present work aims to review the conceptual elements that support the historical construction of a locality; Through theories related with the microhistory and social networks. In the methodology in was proceeded to consult of the National Council of Universities (CNU), referring to books, doctoral theses, and articles related to the study object; this resource was exhausted, and the research was carried out in Google academic. For each subject, several titles were explored, the ideas related to the subject were collected, and the information was analyzed; by author and by concepts, the ideas that contributed to the basis of the research problem were selected. It is concluded that for the construction of the history of a locality, several components of the bibliographic methods must be integrated, such as: the regional and local history, historical memory, social networks, life and oral history, for the rescue of facts, events and their meanings, of a determined period of the society in a territory. It is not only about the knowledge from the past but it also implies the organization of people so that from their individuality they become part of collective thought.

\section{KEYWORDS}

Regional and local history; historical memory; oral history; social networks. 


\section{INTRODUCCIÓN}

Este artículo trata acerca de los fundamentos teóricos relacionados con el proyecto de investigación orientado al estudio del pasado de una localidad', basándose en los postulados que sustenten la construcción histórica de un lugar mediante la historia regional, local y teorías relacionadas a redes sociales. En este artículo se incorporan algunos ejemplos sobre hechos y acontecimientos ocurridos en la ciudad de Chinandega en el periodo de 1960 al 2020, y que serán profundizados en mi tesis doctoral. En dicho estudio se asume el desafío de integrar el protagonismo de los habitantes de una localidad, para que en estos fluyan sus pensamientos, de forma individual y colectiva, por lo que se hace necesario contar con saberes científicos de otras experiencias para que sirvan de referencia para el trabajo investigativo.

En relación con la memoria histórica, Halbwachs,(1968), plantea lo siguiente:

(...) La Historia no es todo el pasado, pero tampoco es todo lo que queda del pasado, o si se quiere, junto a una historia escrita, se encuentra una Historia viva que se perpetúa o se renueva a través del tiempo y donde es posible encontrar un gran número de esas corrientes antiguas que sólo aparentemente habían desaparecido. (...) Halbwchs. (p.1).

Es importante aclarar, que como parte de los criterios para la selección de los informantes se requiere que sean personas entre los 60 o 70 años, ya que les ha correspondido vivir esta etapa en sus vidas.

Thompson, (2004), plantea algunas consideraciones en relación con la Historia oral:

En primer lugar, decididamente me inclino por una definición amplia de "Historia oral" como la interpretación de la Historia, las sociedades y las culturas en proceso de cambio a través de la escucha y registro de las memorias y experiencias de sus protagonistas, y por lo tanto no me parece apropiado concebirla ni como un método de trabajo minuciosamente determinado y con reglas fijas ni como una su disciplina separada.(p.15).

Al trabajar la historia con una filosofía flexible para interpretar distintos aspectos cambiantes de una sociedad, en temas actuales de diferentes esferas de la vida de la gente, referido a hechos y acontecimientos que acontecieron en el pasado de un país, una región o una localidad, sea esta: ciudad, pueblo, villa, barrio, colonia reparto, comarca o caserío; mediante los diálogos enfatizados en la escucha, registro de los recuerdos y experiencias personales de quienes cuentan su Historia, que generen resultados con una mayor riqueza de saberes, debido a esa amplitud que facilita al tomar

1 Este artículo se enmarca en la revisión teórica de mi investigación de doctorado titulada: Redes sociales como modelo metodológico para la construcción de los significados de la memoria histórica local: Chinandega 1960- 
la información de distintas fuentes, lo que conlleva una visión amplia del pasado de esta misma sociedad.

Hay que destacar que los objetivos del trabajo están orientados a: revisar los elementos teóricos y conceptuales que responden al método bibliográfico vinculados a la Historia: regional, local, oral y memoria histórica; desde un enfoque constructivista. Así como determinar las principales concepciones teóricas de un modelo basado en redes sociales, entendido como una interconexión de personas que actúan en diferentes escenarios tales como comunitarios, sociales, gremiales y otros.

\section{MATERIALES Y MÉTODOS}

El presente estudio responde a una investigación de carácter documental realizada para el curso de redacción científica del doctorado de la gestión y calidad de la investigación científica que se desarrolla en la Facultad multidisciplinaria de Estelí. (FAREM-Estelí) de la Universidad Nacional Autónoma de Nicaragua (UNAN-Managua, acerca de referentes teóricos relacionados con la historia regional y local, memoria histórica, historia de vida, historia oral y redes sociales, para este fin se procedió de la siguiente manera: se definieron los objetivos de este trabajo investigativo, se adaptó matriz de recopilación de datos facilitada por la facilitadora del curso redacción científica que se realizó en el doctorado y se determinó un orden de la temática, para la búsqueda de información de esta forma: historia regional y local, memoria histórica, historia oral, historia de vida y redes sociales; luego se procedió a la consulta del repositorio de la UNANManagua en la búsqueda de libros, tesis doctorales y artículos vinculados al objeto de la búsqueda. Agotado este recurso se procedió a la búsqueda en Google académico.

Para cada tópico, se exploraron y revisaron los documentos para leer, luego seleccionar las ideas relacionadas a la temática en indagación, ya obtenido el resultado positivo se procedió a copiar en la matriz de análisis de datos las partes de interés. Culminado el proceso de indagación, se realizó el análisis de la información; en primer lugar, por autor y en segundo lugar por conceptos de la misma temática. Para cada conceptualización se fueron seleccionando las ideas que sirviesen de fundamento relacionado al problema de investigación que tiene que ver con los elementos teóricos que lo sustenten. Posteriormente se procedió a redacción de los resultados que permitan la discusión. 
En el proceso de indagación se utilizaron como medios para llegar a las fuentes los repositorios de la UNAN-Managua, UNAN-León y el buscador Google académico, por medio de los cuales se consultó a autores que han trabajado aspectos teóricos concerniente al objetivo de esta investigación. En correspondencia a los criterios de selección de la información se determinaron los siguientes: información de tesis doctorales, libros, artículos y ensayos científicos. documentación concerniente a la historia regional y local. Datos acerca de memoria histórica, planteamientos relacionados a la historia oral y de vidas. Así como teorías de redes sociales.

\section{RESULTADOS}

La construcción de la memoria histórica de una localidad requiere de un cuerpo teórico y procedimental sustentado por diferentes concepciones, en su mayoría complementan entre ellas, esto le permite al investigador tener una variedad de postulados que puede aplicar acorde al paradigma en cual se identifica.

En este caso se retoman conceptos asociados al paradigma Constructivista, ya que se involucra a las personas en la recuperación de hechos, acontecimientos que han ocurrido en un pasado cercano que ha correspondido vivir y por otra parte puedan explicarse los significados que la gente da a estos. Se trata de generar un modelo que facilite la participación e involucramiento de la población desde las comunidades, con el apoyo de los postulados de la historia regional, local y redes sociales desde sus aportes conceptuales y metodológicos contribuyen en la búsqueda y construcción de conocimientos teóricos.

El investigador debe orientar su quehacer en producir conocimientos que tengan un mayor aprovechamiento de la sociedad, es decir que no sea simplemente una recolección de datos históricos, sino que lo indagado permita retomar aquellas experiencias que contribuyan a alcanzar mayores niveles de desarrollo a la sociedad estudiada. Al tratarse de la historia de una localidad con la participación de la población, habrá una mayor proximidad para su utilización en función de esta.

Vera, (2014), considera que hay que tomar en cuenta el papel de la historia, ya sea como ciencia humana o como una ciencia que responde a las ciencias sociales. Debe explicar la realidad de la sociedad enfatizando en las personas, estimular la memoria hacia hechos y acontecimientos que ocurrieron en el pasado o bien generar en la sociedad emociones y sentimientos de identidad. Esta puede relacionarse a su etnia, cosmovisión, costumbres, tradiciones, causa social o política, estilo de vida y a la territorialidad, que tenga como propósito contribuir en la producción de saberes que expresen significados para los habitantes a nivel micro o macro, según sea el estudio que se realice. 


\section{Historia Regional y local}

Se trata de construir la experiencia vivida de una sociedad mediante la historia local y regional como un método histórico que cuenta con fundamentos teóricos y metodológicos para guiar y orientan el proceso de generar el conocimiento acerca del pasado de esta sociedad en sus diferentes esferas.

Reina (1993) plantea "la región histórica abarca lo que se refiere a aspectos demográficos y vínculos de estratos sociales" (p. 138). Los aspectos demográficos ayudan a entender la dinámica de una población que vive en un territorio pequeño que tiene su identidad particular; en cuanto al tema de los estratos sociales, en nuestra sociedad contemporánea, clases sociales, analizar cómo interactúan en el entorno geográfico teniendo como referente la posesión sobre los medios de producción e instrumentos de trabajo. Tanto el aspecto demográfico como las clases sociales intervienen en la configuración de una localidad.

El territorio como una unidad que permite ser sujeta de análisis puede entenderse con diferentes magnitudes, en este caso interesa la relación existente entre la definición de una región histórica como el pasado de una localidad; en relación a los aspectos teóricos son ajustables y en cuanto al tratamiento como una unidad de análisis, las variables pueden ser aplicadas en un espectro regional o local; Reina,(1993), enfatiza "La región es una unidad de análisis con características estructurales propias y delimitada por el conjunto de relaciones sociales que establecen sus grupos sociales en un espacio y en un periodo determinado" (p.138).

Por otra parte, Venegas (2010) en su contribución a la historia regional y local, hace referencia a la variable urbana; en determinadas circunstancias se convierte en el centro de una región o de una localidad cuando es delimitado para ser estudiado desde historia regional y local, al respecto señala: "Este sistema urbano y semiurbano muchas veces también- es el que centra y focaliza toda la vida regional, incluyendo la rural, lo que se ahonda en el transcurso y progresión del tiempo histórico regional. (...)" (p. 67)

La sociedad vive un proceso evolutivo permanente, esos cambios y transformaciones se vienen generando en dos vías: cambios a nivel macro y otros a nivel micro, ambos provocan efectos, los grandes cambios que pueden abarcar varios países o el mundo inciden en una localidad; los cambios que inician desde lo micro en alguna medida van ampliando su ámbito de transformación de una localidad a una región o bien todo un país. Molina, (2013), "señala que provocan cambios en ámbito socio geográficos de mayores dimensiones. o viceversa los cambios macro de una región o país inciden en los cambios de una localidad." (p.22).

Las tradiciones de la sociedad forman parte de los elementos que deben tomarse en cuenta en la construcción de la historia de una localidad, en estas 
el investigador debe desarrollar su estudio en dos dimensiones: en primer lugar en lo que respecto al origen cronológico de las tradición y en segundo lugar el aspecto que se relaciona con los significados de cada una de ellas; estos significados pueden ser distintos entre las personas de una misma generación o bien de otra generación anterior o posterior, Molina,(2013) señala lo siguiente "La práctica de la historia local generalmente obliga al historiados a considerar en el problema de la investigación, lo tradicional" (p.24)

La definición más clara de historia regional o local, según Serrano, (2009), plantea dos variables a tomar en consideración: un primer aspecto se refiere a la existencia de una delimitación de un área geográfica concreta, en la cual interactúa una sociedad en sus diferentes esferas tales como: la social, económica, política y cultural entre otras y en segundo lugar es la consideración de una temporalidad definida, es decir durante que años se realizará el estudio histórico. En esto entorno existe una identidad que la hace diferenciarse de otras unidades en las que interactúan sociedades en un territorio específico.

El surgimiento de la propuesta de un estudio apoyándose en la región histórica, está dado por los elementos del territorio, ya sea este una región histórica o una localidad, Serrano, (2009), plantea que debe de tomarse en cuenta los siguientes elementos en la realización de un estudio regional o local: las relaciones sociales, la temporalidad, la identidad, y la totalidad, sean estos en diferentes ámbitos de la dinámica de una sociedad.

Para la historia regional y local, el ámbito de la localidad como una unidad de análisis para construir la microhistoria de una población determinada, distintos autores señalan que el investigador debe considerar algunas variables en este tipo de trabajo, entre las cuales están las siguientes: área geográfica, delimitación temporal, demografía, estratos sociales tradiciones, desarrollo urbano, identidad, desarrollo humano entre otros. A pesar de la existencia de una variedad de enfoques y métodos se debe trabajar, tomando en cuenta que no todo está dicho por lo que hay que enfrentar problemas y limitaciones a la cuales habrá que darle respuesta según el estudio a realizar.

\section{Redes sociales}

Para la construcción de la historia de la localidad desde el paradigma constructivista se requiere involucrar al mayor número de habitantes que conocen de los hechos y acontecimientos a construir mediante la memoria histórica e historia oral, para ellos es importante destacar elementos conceptuales relacionados a redes sociales.

Lozares, (1996), establece varias definiciones sobre este concepto, en este caso interesa la idea de redes sociales asociada a personas en su condición individual, de grupo, estos últimos agrupados como vecinos, comunidad, 
pueblos, asociaciones de distintas características en su ámbito y su dinámica ya sea esta: social, religiosa, artística, laboral, deportiva entre otros, que comparten intereses y objetivos comunes. En el caso de la historia de una localidad recuperada mediante la memoria histórica.

Quesada, (1993), citando a (Barnes, 1954) y (Mitchel 1969) define a una red social como "un conjunto concreto de personas de forma particular de conexiones e interacciones entre ellos, en la cual sus características son comunes, lo que permite interpretar el protagonismo en desarrollo de eventos sociales, económicos, productivos entre otros". Partiendo de estas conceptualizaciones de redes sociales vinculadas al paradigma constructivista, son las bases para el desarrollo del trabajo investigativo, ya que este se orienta a un modelo del protagonismo de sus habitantes, en la construcción de la historia de su localidad.

El estudio que utilice las concepciones de redes sociales relacionado a la memoria histórica de una localidad, debe entenderse como un conjunto de conexiones entre personas y colectividades que poseen características similares tales como: haber vivido el mismo periodo que abarca el estudio, actividades realizadas en común en lo económico, artístico, deportivo, festivo, conmemoraciones, participación política entre otros, esta interacción puede darse al encontrarse personas de distintos lugares del municipio, así mismo esta red involucra a personas que provienen de una misma comunidad y en representación de esta participan con otros representantes que pertenecen a otros lugares del mismo municipio.

\section{De la memoria histórica}

Se trata de establecer un vínculo entre la memoria de la persona en relación con hechos y acontecimientos históricos que han sucedido en un tiempo claramente definido y que guarda en sus recuerdos una persona, al establecer las ideas entre la gente, da lugar a la confrontación de información sobre un mismo asunto, no solo observar esas contradicciones, sino que formar parte de una solución.

La conceptualización de memoria colectiva ha sido objeto de ser explicada con distintos términos, que no aportan para la formulación de una interpretación única, ya que algunos estudios la representan como símbolos como fuentes que deben ser analizadas y conservadas por una sociedad a través del tiempo. Dada esta situación la memoria colectiva se utiliza como emblemas que sirven como fuentes, así mismo otra parte plantean su preocupación orientada hacia los procesos, partiendo que estas representaciones se edifican y mantienen en el tiempo tales como monumentos y celebraciones conmemorativas.

Una investigación basada en la memoria colectiva, debe ponerse atención a varios procesos, tomando en consideración: transmisión de recuerdo, para 
promover, convergencia de estos, estabilidad de aquellos pensamientos que formante parte no solo de una persona, sino de una colectividad de una misma temporalidad. Asociados con aspectos que los identifica como parte de una misma sociedad con objetivos e intereses comunes. Las conversaciones sobre hechos y acontecimientos que ocurrieron en el pasado se convierte en una forma que permite las transferencias de lo ocurrido, de una persona hacia otra. El tema del contagio social favorece que las evocaciones individuales se vayan convirtiendo en rememoraciones colectivas.

Según Albwachs, (1998), identifica dos tipos de recuerdos asociados a la memoria colectiva: los pensamientos guardados que se relacionan con experiencias vividas o bien los que se relacionan a su vida $y$, por otra parte, otras memorizaciones que tienen que ver con hechos y acontecimientos históricos que ocurrieron en determinado tiempo. Las experiencias vividas sirven como fuente a la experiencia individual que una persona tiene acerca de un determinado evento ocurrido en el pasado, este tipo de pensamiento en relación al pasado, tiende a desaparecer con el tiempo, si no es compartido y comunicado, en conjunto con otros participantes que formaron parte del mismo acontecimiento. Este tipo de información se encuentra enraizada en la persona.

Müller y Bermejo (2013) citando a Schuman y Scott (1999) consideran que se hace necesario tener en cuenta dos definiciones de memoria colectiva, una que se apoyan entre la distinción entre recuerdos vividos y la otra que refiere a los recuerdos históricos cuando una parte representativa de la población recurre a sus pensamientos del pasado de un acontecimiento en común, por ejemplo, la masacre del 2 y 3 de junio de 1979 en Chinandega, Nicaragua, este recuerdo pasa a formar parte de la memoria colectiva. Esta memoria colectiva tiene dos fuentes: la de aquellas personas que conocieron directamente de hechos y la otra fuente cuando año con año se realiza una conmemoración de este hecho histórico y queda en las ideas de quienes no lo experimentaron de forma directa.

Por ejemplo, en la actividad económica en los años 60 del siglo XX, en el municipio de Chinandega, donde se enfocará mi tesis doctoral, predominaban dos actividades productivas agrícolas: en primer lugar el cultivo del algodón y en segundo el cultivo del banano, en relación al primero con el tema del reforzamiento de los recuerdos hay centenares de habitantes de este municipio que participaron directamente en esta actividad desde la preparación de la tierra, siembra, cuido y corte de la mota blanca, si se delimita a una población que participó en misma hacienda algodonera los recuerdos serán específicos de este lugar y puede ser reforzados por otras personas que también estuvieron ahí, al tomarse una muestra de mayor tamaño que involucre a gente que participó en diferentes lugares, también pueden reforzar esos recuerdos. 
Un recuerdo individual, que es parte de una sociedad, refleja el hecho o acontecimiento según la experiencia y circunstancia en que le correspondió vivir a esta persona, al darse la interacción con otras personas la misma experiencia guardada en la memoria desde lo individual, reforzada ya que con los recuerdos de otros, afloran nuevos recuerdos que no habían sido actividades en su cerebro, ahora bien lo colectivo apoya a lo individual, pero también, lo individual enriquece a la memoria colectiva, Entre más personas se involucren en la construcción del pasado sus versiones van adquiriendo una mayor consistencia y confianza.

Según Albwachs (1968), la persona conforme su edad, va adquiriendo una participación progresiva que se va ir diferenciando y será más reflexiva en la vida e ideas de los grupos, a medida que la persona atraviesa una nueva etapa de su vida: niño, adolescente, joven, adulto; los niveles de integración y construcción de memoria irán madurando y adquiriendo mayor información, para cada etapa de su vida la persona va construyendo recuerdos que le son de su experiencia personal o bien pertenecen a recuerdos creados a través de los grupos.

Según, Ricoeur, (1999) en el abordaje de la memoria de ciertos hechos que no tiene una salida lógica en relación con el problema de la memoria, en sus planteamientos están los siguientes:

La primera se refiere a la difícil conciliación del tratamiento de la memoria como experiencia eminentemente privada, e interna con su caracterización como fenómeno social colectivo y público. La segunda se refiere la relación que existe entre la imaginación, en cuanto a la función de la ausencia de huellas temporales, y la memoria (...). La tercera se refiere al derecho a introducir consideraciones (...); la relación que existe entre la memoria y la construcción de la identidad personal o colectiva (...) ( p. 13,14).

La experiencia de lo privado y lo de la imaginación son dos retos a conciliarse en relación al tratamiento de la memoria; hay recuerdos de las personas que corresponde a su vida privada es decir, no interesa al resto de la población, pero hay recuerdos del actuar de esta persona que no tienen que ver con lo íntimo, sino que forman parte de una construcción e interés social, esta parte es la de interés de la memoria histórica, aquellos pensamientos que forman parte para construirlos de forma colectiva. En lo corresponde a la imaginación es la parte subjetiva ya que las evidencias o registro de la imagen de ese recuerdo no pueden guardar la fidelidad tal y como sucedió en el pasado.

Siguiendo con el ejemplo de la región de Chinandega, cuando una persona entre los años setenta y ochenta del siglo $\mathrm{xx}$, que viajaba en tren entre Chinandega y León, sus recuerdos de esa época pierden de alguna manera fidelidad, como se trata de una construcción colectiva esas imágenes que sea fieles a la época pueden ser reforzada por otras personas que vivieron la misma experiencia al ser compartidas de forma colectiva 


\section{Historia oral}

El trabajo por realizar busca una fundamentación teórica que ayude a responder el trabajo investigativo a través de la Historia oral, al destacar los aportes conceptuales de: Paul Thompson y Laura Benadiba que figuran entre algunos de los representantes de este método.

Se trata de la interpretación de aquellos hechos y acontecimientos históricos que se necesita de la expresión oral del que pregunta y escucha de lo que se pretende saber en relación con su objeto de investigación y de quien contará su experiencia relacionado al tema en estudio, hay un diálogo en el cuál debe predominar la intervención del entrevistado, este diálogo debe responder a algo planificado con anticipación, sin embargo es importante tomar en cuenta que puede ser ajustado en el desarrollo de la entrevista o con otra persona entrevistada acerca del mismo tópico.

Thompson, (2004), plantea que deben combinarse resultados cualitativos y cuantitativos para obtener una mejor investigación, implica descifrar aspectos individuales de cada persona en lo individual y de carácter social que produzcan explicaciones con significados y datos estadísticos que complemente al abordaje del tema en estudio, esto contribuye a una mejor explicación del objeto de estudio, así mismo el investigador debe auxiliarse de otros métodos, técnicas e instrumentos y fuentes para obtener información complementaria. En relación con esa combinación se plantea lo siguiente:

La mejor investigación de historia oral es aquella que abarca tanto la comprensión e interpretación de vidas individuales como un análisis social más amplio, en otras palabras, la que articula los resultados de la investigación cualitativa con los de la investigación cuantitativa. (Thompson,2004, p .19)

Al realizar investigaciones, aplicando la metodología de la historia oral se debe de tener el cuidado de no presionar a la persona entrevistada para obtener ideas que queremos escuchar y no las que él quiere expresar, él interpretar cambios sociales implica que en la metódica se seleccione una muestra representativa y cumpla con los criterios para obtener resultados de calidad. Es recomendable que se tome en cuenta al seleccionar a los entrevistados que estos pertenezcan a más de dos generaciones, es decir que tengan diferentes edades.

Thompson, (2004), hace referencia de la existencia de algunas temáticas que le dan fuerza a la historia oral, lo que permite agruparla en cuatro tópicos: voces ocultas, esferas escondidas, tradición oral y establecimiento de conexiones entre vidas. De estas cuatro temáticas, destaca que cada persona sea hombre o mujer posee una historia de vida histórica y que interesa a la sociedad, ya que tradicionalmente, solo se conocen las voces de personas influyentes de la sociedad; A través de esta le permite el acceso para poder expresar y evidenciar una Historia que no ha sido contado. 
En su abordaje de las esferas escondidas, Thompson, (2004), se refiere a "aquellas historias que conocen personas comunes y que sus relatos no están registrados como parte de la historia de la sociedad" ( p.22), las considera como las de mayor importancia en todas las relaciones familiares de una sociedad, hay muchos acontecimientos y hechos históricos que han ocurrido en el pasado, que, a pesar de no estar en los registros oficiales de la Historia, tienen significado y relevancia en las familias.

Thompson (2004), señala otras esferas escondidas tales como: la descomposición asociadas a él crimen, la violencia y las drogas, es decir en cualquier espacio geográfico y social se pueden investigar y desarrollar estas temáticas para entender algunas historias de vida; otra esfera desapercibida es la cultura informal del trabajo, en los países subdesarrollados la pequeña y mediana economía formal y no forman aportan sustancialmente al desarrollo de un país, sin embargo en los registros de la historia económica quedan ignorados ante la relevancia que le dan a los que aglutinan mayores riquezas, en estos sectores informales se desarrolla una riqueza de saberes que se manifiesta en su vida cotidiana.

La historia oral viene adaptándose a las circunstancias contemporáneas dominadas por el uso de las tecnologías de la comunicación, en los últimos años los cambios en relación al uso del sonido se han modificado enormemente, lo que se convierte un retos para los historiadores en ajustar el uso de recursos acordes con estos nuevos tiempos, esta condición se proyecta no como una debilidad sino como una oportunidad para recolectar información y publicarla, estos cambios se presentan como una oportunidad para la historia oral.

Benadiba, (2015), señala que la historia oral es un recurso valioso para poder registrar de forma escrita, hechos y acontecimientos que ocurrieron en un pasado, que sirve como punto de encuentros entre varias disciplinas, contando para esto con personas que aún están vivas y se vieron involucrados de distintas formas, fueron parte protagónica en la historia o bien como observadores poseen la misma información y saberes para documentar y dejar evidencia de la existencia de un periodo de la historia, cercano a la persona que relata el hechos o acontecimiento. Desde su perspectiva plantea los siguiente:

(...) podemos definir la historia oral como un procedimiento establecido para la construcción de nuevas fuentes para la investigación histórica, basándose en testimonios orales recogidos sistemáticamente a través de entrevistas, a partir de métodos, problemas y parámetros teóricos concretos. (...), su utilización sistemática y reflexiva está asociada al desarrollo de la historia social contemporánea, más preocupada, en general, por los procesos sociales y por la vida de la gente "común" (Benadiba,2015, p.91).

En las ideas expresadas por Benadiba, (2015), precisa que desde la historia oral se hace una contribución importante a la investigación histórica, entre 
sus aportes se pueden mencionar: sirven de complemento a las fuentes que se han usado de forma tradicional, en relación a los significados de los hechos y acontecimientos presenta una mayor precisión, pues permite que a través de sus expresiones los informantes resaltan aspectos que no se aprecia en una fuente escrita, desde la práctica, permite un cuestionamiento crítico del pasado vinculando con su presente, permite la construcción del historia por medio de gente que tiene una vida cotidiana igual que el resto de la gente.

Benadiba (2007). reflexiona acerca del riesgo de olvidar aquellas partes de la historia que no encuentran están escritas, sino que se guardadas en la memoria de la persona, cuando se da el fenómeno de no transferir estos saberes de una generación a otra, es decir la generación antecesora no trasladar sus experiencias y los de sus antepasados, a la generación sucesora.

Benadiba, (2007), establece una relación entre memoria e historia oral, esto permite obtener pruebas históricas; a partir de esas experiencias individuales o colectivas se generan elementos para el análisis e interpretación de sucesos históricos a partir de la producción de fuentes escritas para demostrar su condición de veracidad, las cuales respaldan el significado de lo ocurrido y le dan validez científica al estudio realizado al producir fuentes.

\section{CONCLUSIONES}

La revisión documental realizada permitió el cumplimiento de los objetivos de este trabajo, ya que revisaron aquellos elementos teóricos del enfoque bibliográfico y redes sociales que se necesitan para la construcción de la memoria histórica de una localidad. El papel de la historia y el historiador es producir conocimiento que conlleve a su utilización en el presente inmediato, por lo que debe explicar la realidad humana de los ocurrido en un periodo determinado.

La recuperación de la historia de una localidad, mediante la participación de su población cuenta y puede integrar varios métodos del enfoque bibliográfico, tales como la historia regional, local, de vidas y oral, memoria histórica, redes sociales, mediante los cuales se rescatan hechos y los significados de un período de la historia de una sociedad en la que la ha correspondido vivir.

En el estudio de una región o localidad se deben tomar en cuenta determinadas condiciones que sirvan de plataforma como base una delimitación geográfica en la que han interactuado seres humanos en periodo determinado, ambas son unidades de análisis para que el investigador pueda examinar una variedad de variables tales como: demografía, relaciones sociales, actividades 
económicas, organización político-administrativa, desarrollo urbano y rural, tradiciones, arte, deporte entre otros.

La historia regional y local, a pesar de contar con una variedad de enfoques y métodos, por su misma diversidad y flexibilidad, presentan algunas limitaciones y problemas que se deben dar respuesta según el estudio que se realice.

En relación de las redes sociales la definición que se corresponde con el objeto de investigación se asume como personas que en su condición individual o de grupo, establecen una gran variedad de conexiones, producto de dinámica en la vida cotidiana establecen contacto como familia, vecinos, compañeros de trabajo, actividades religiosas, actividades deportivas, en uso de las tecnologías de la comunicación, cooperativas, sindicatos, asociaciones gremiales entre otros.

Indiscutiblemente las concepciones de la memoria histórica y la historia Oral se convierten en un recurso valioso en la producción de conocimiento histórico de aquellos hechos y acontecimientos que carecen de evidencia para sustentar su veracidad, este sentido el término que tiene una mayor aproximación son aquellos recuerdos relacionados al pasado histórico de una sociedad. Por ello al trabajar con la memoria histórica requiere de prestar atención a ciertos procesos tales como la transmisión, del recuerdo, el contagio social, la transición de un recuerdo individual a un pensamiento colectivo. Aunque algunos estudios se han hecho para expresar los traumas producto de conflictos políticos, sociales, el método de la memoria histórica se puede aprovechar para que la gente exprese su pasado local, principalmente aquellas que van marcando sus transformaciones y desarrollo en todas las esferas de su sociedad.

En los recuerdos de ese pasado el historiador debe clarificar los que son el resultado de experiencias vividas en lo personal y las memorizaciones de hechos y acontecimientos que se obtienen de otras formas tales como la escuela, libros, tradición oral entre otros. Para la construcción de la memoria histórica de una localidad tenga una mayor solidez cuando se involucran más personas, en la construcción de un pasado común.

Existe una estrecha relación al trabajar los métodos de la memoria histórica y la historia oral debido a lo siguiente: para que una persona exprese sus recuerdos, requiere de la escucha y el registro de lo expresado, es decir que para que esos recuerdos históricos salgan de la memoria, debe haber una diálogo mediado por una entrevista, campo que corresponde a la historia Oral, la cual se define como la interpretación de la historia, las sociedades y la cultura a través de la escucha y registro datos cualitativos y cuantitativo, en la obtención estos resultados se debe de tener el cuidado de inducir a respuestas que son propias del entrevistador y no del informante. 
De ahí que al desarrollar un trabajo de historia local, con una concepción constructivista que no solo interesen los datos e información histórico, sino que también los significados que le da la gente a su pasado vivido, de ahí que la construcción de la memoria histórica de una localidad debe conjugar la aplicación de varios métodos del enfoque bibliográfica, que respondan a un proyecto investigativo en el cual el objeto de estudio es un pasado reciente, en cual es indispensable que los informantes hayan vivido y formado parte de la experiencia a indagar.

La participación de la población requiere que sea mediante un enfoque de redes sociales, en el que se parte de una individualidad, que, en sus conexiones variadas, se van formando redes de personas que interactúan en función de distintos objetivos e intereses en un entramado territorial, social y virtual, formando comunidades que comparten una Historia local en común y ayuda a la recolección de datos, contrastación de la información para darle una mayor credibilidad a los resultados obtenidos.

\section{REFERENCIAS BIBLIOGRÁFICAS}

Benadiba, L. (2005) introducción a la Historia Oral recuperado de:https:// es.scribd.com/document/364545151/De-Entrevistadores-y-Relatos-deVida-Introduccion-a-La-Historia-Oral

Benadiba, L. (2009), Historia oral, relatos y memorias. CONHISREMI, Revista Universitaria de Investigación y Diálogo Académico. Vol. 5, No. 1, recuperado de:https://www.comisionporlamemoria.org/archivos/ archivo/archivo-oral/bibliografia/Historia\%20oral, \%20relatos\%20y\%20 memoria_introduccion.pdf

Chárriez Cōrdero, M. (2012) (1). Historias de vida: Una metodología de investigación cualitativa. Revista Griot, 5(1), 50-67. Recuperado a partir de https://revistas.upr.edu/index.php/griot/article/view/1775

Gailondo, Á. (1999). Lectura del tiempo pasado: Memoria y olvido. Recuperado de https://www.academia.edu/23969014/Ricoeur_La_ lectura_del_tiempo_pasado_Memoria_y_Olvido?auto=download

González, Matias and Montanares, Elizabeth and Martínez, Francisca, (2018): Estudio reflexivo para abordar la Historia local en Chile desde la versión anglosajona (A Reflective Study to Approach the Local Chilean History from the Anglo-Saxon Perspective) (January 1, 2018). HiSTOReLo. Revista de Historia Regional y Local, Vol. 10, No. 19, enero - junio de 2018, Available at SSRN: https://ssrn.com/abstract=3139959 or http:// dx.doi.org/10.2139/ssrn.3139959p

Halbwachs, M. \& Díaz, A. (1995). Memoria colectiva y memoria histórica. Reis, (69), 209-219. doi:10.2307/40183784. Recuperado de: https:// www.jstor.org/stable/40183784 
Lozares, Colina, C. (1996). Teoría de las redes sociales, Las redes sociales. Revista Papers, no.48 recuperado de https://ddd.uab.cat/ record $/ 53049$ ? $\mathrm{ln}=$ en\& of $=\mathrm{hm}$

Molina, Jiménez, I, (1996). De la Historia Local a la Historia social: algunas notas metodológicas. Volumen51 no. 1 recuperadode:https://revistas.ucr. ac.cr/index.php/reflexiones/article/view/10926 21/22

Muller, Mermejo. (2013). Fuentes de las memorias Colectivas: los recuerdos vividos e históricos. Revisat de Psicología volumen no.2.recuperado de:http://www.scielo.org.pe/scielo.php? pid=S025492472013000200004\&script $=$ sci_arttext

Ponce, A. (,2008), Redes sociales y ejercicio del poder en la América Hispana: consideraciones teóricas y propuestas de análisis 1, Revista Complutense de Historia de América, vol.34,15-42P.P recuperado:https://revistas.ucm. es/index.php/RCHA/article/download/RCHA0808110015A/28432

Reina, L. (1992). Historia Regional e Historia nacional. Revista Historias, estudios historicos.inah.gob.mx,recuperadode:https://www. estudioshistoricos.inah.gob.mx/revistaHistorias/wpcontent/uploads/ Historias 29 131-142.pdf

Ricoeur,P (1999). Lectura del tiempo: Memoria y Olvido, Recuperado de https://www.academia.edu/23969014/Ricoeur_La_lectura_del_tiempo_ pasado_Memoria_y Olvido?auto=downloadISBN̄ $: \overline{4}-9237 \overline{9} 2-2-7$

Serrano Álvarez,P. (2009). La Historia local en América Latina Tendencias, corrientes y perspectivas en el siglo XX Historelo.rev.hist.reg.loca I vol.1 no.1 Medellín Jan. /June 2009 Tomado de: http://www.scielo.org.co/ scielo.php? script $=$ sci_arttext\&pid $=$ S2 145-132X2009000100002

Thompson, P. (2004) $\dot{2}$ Historia oral y contemporaneidad, recuperado de:http:// biblioteca.puntoedu.edu.ar/bitstream/handle/2133/1 1963/204-353-1 PB.pdf? sequence $=2$ \&isAllowed $=y$

Venegas, H. (2010), Metodología de la investigación en Historia Regional y Local, Archivo General de la Nación Volumen XCl, recuperado de Descargar - Archivo General de la Nación (yumpu.com) ISBN 978-9945020-78-6

Vera, Jaramillo, J. (2014), Eric Hobsbawm, Revista Divergencia n. 16, marzo de 2014 | Universidad Externado de Colombia: recuperado de: https:// core.ac.uk/download/pdf/230103385.pdf revista p,70

Villalta Quezada, C. (1993) Redes Sociales: Un concepto con importantes implicaciones en la intervención comunitariahttp://www.copmadrid.org/ webcopm/publicaciones/social/1993/vol1/arti6.htm 